\title{
ASPECTOS BIOLÓGICOS Y PARÁMETROS POBLACIONALES DE Cycloneda sanguinea L. (COLEOPTERA: COCCINELLIDAE) SOBRE Aphis aurantii BOYER DE FONSCOLOMBE (HEMIPTERA: APHIDIDAE)
}

\author{
BIOLOGICAL ASPECTS AND POPULATION PARAMETERS OF Cycloneda \\ sanguinea L. (COLEOPTERA: COCCINELLIDAE) ON Aphis aurantii BOYER \\ DE FONSCOLOMBE (HEMIPTERA: APHIDIDAE)
}

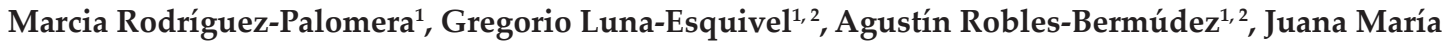
Coronado-Blanco ${ }^{3}$, Kevin Gerardo Cambero-Nava ${ }^{4}$, y Octavio Jhonathan Cambero-Campos ${ }^{1,2 *}$

${ }^{1}$ Programa de Doctorado en Ciencias Biológico Agropecuarias, Universidad Autónoma de Nayarit, Carretera Tepic-Compostela Km. 9. C.P. 63155, Xalisco, Nayarit, México.

${ }^{2}$ Unidad Académica de Agricultura, Universidad Autónoma de Nayarit, Carretera Tepic-Compostela Km. 9. Xalisco, Nayarit, México.

${ }^{3}$ Facultad de Ingeniería y Ciencias, Universidad Autónoma de Tamaulipas, Centro Universitario Adolfo López Mateos, C.P. 87149. Cd. Victoria, Tamaulipas, México.

${ }^{4}$ Centro Universitario de la Costa, Universidad de Guadalajara, Av. Universidad 203. Delegación Ixtapa. C.P. 48280. Puerto Vallarta, Jalisco, México.

*Autor para correspondencia E-mail: jhony695@gmail.com

\section{RESUMEN}

En el presente trabajo se determinó el ciclo biológico y tabla de vida de Cycloneda sanguinea $\mathrm{L}$. alimentado con el pulgón Aphis aurantii Boyer de Fonscolombe en condiciones de laboratorio. El material biológico se recolectó en huertas de yaca (Artocarpus heterophyllus Lam.) ubicadas en los municipios de San Blas y Compostela, Nayarit, México. Los experimentos se llevaron a cabo en el Laboratorio de Parasitología de la Universidad Autónoma de Nayarit, a una temperatura de $25 \pm 2{ }^{\circ} \mathrm{C}$, $70 \pm 10 \%$ HR y una fotofase de 12 horas. En estas condiciones, la duración de la fase inmadura fue de $19,32 \pm 4,97$ días y la longevidad de los adultos de 58,37 $\pm 3,44$ y 61,71 $\pm 4,96$ días, para machos y hembras, respectivamente. Las hembras presentaron un periodo de pre-oviposición de 3,9 $\pm 0,31$ días, con una fecundidad de $882,2 \pm 52,28$ huevos/hembra. La curva de sobrevivencia fue de tipo I, en donde la mortalidad se concentró en los individuos de edades avanzadas, mientras que la curva de fecundidad, mostró dos puntos máximos de oviposición en los días 9 y 36 después de la emergencia de los adultos. La tasa neta reproductiva fue de 705,76 huevos hembra/hembra, en un tiempo generacional de 23,39 días y una tasa finita de crecimiento de 1,41 individuos por día.

Palabras clave: Control biológico, pulgón negro de los cítricos, depredadores, yaca.

\section{ABSTRACT}

The objective of this study was to determine the biological cycle and life table of Cycloneda sanguinea L. fed with the aphid Aphis aurantii Boyer de Fonscolombe under laboratory conditions. The biological material was collected in jackfruit (Artocarpus heterophyllus Lam.) orchards located in the municipalities of San Blas and Compostela, Nayarit, Mexico. The experiments were carried out in the Laboratory of Parasitology of the Autonomous University of Nayarit, at a temperature of $25 \pm 2{ }^{\circ} \mathrm{C}$, $70 \pm 10 \%$ RH and a photophase of 12 hours. The duration of the immature phase was $19.32 \pm 4.97$ days, 
and the longevity of the adults was $58.37 \pm 3.44$ and $61.71 \pm 4.96$ days, for males and females, respectively. The females presented a pre-oviposition period of $3.9 \pm 0.31$ days, with a fecundity of $882.2 \pm$ 52.28 eggs / female. The survival curve was type I, which indicated that mortality mostly occurred in old individuals. The fertility curve showed two maximum oviposition peaks on days 9 and 36 after the emergence of adults. The net reproductive rate was 705.76 female eggs/female, in a generational time of 23.39 days, and a finite rate of increase of 1.41 individuals per day.

Key words: Biological control, black citrus aphid, predators, jackfruit.

\section{INTRODUCCIÓN}

La familia Coccinellidae es uno de los grupos del orden Coleoptera con mayor importancia ecológica, debido a la constante actividad depredadora que ejercen larvas y adultos sobre sus presas; algunas especies son micófagas, otras tienen hábitos fitófagos, y existen, además, especies que se pueden alimentar de polen (Castro, 2017). En México, López et al. (2007) registraron 87 especies de coccinélidos depredadores con potencial para el control de plagas de importancia agrícola; las especies consumidoras de áfidos (Hemiptera: Aphididae) son consideradas como los depredadores de mayor conocimiento taxonómico, y su estudio se le asocia únicamente al cultivo y el rango de presas consumidas.

De acuerdo a Vandenberg (2002), por su distribución cosmopolita, Cycloneda sanguinea L. es una de las especies mejor conocidas dentro de los Coccinellidae, y es catalogada como uno de los agentes de control más eficientes de insectos fitófagos estacionarios como los pulgones (Funichello et al., 2012). Su presencia ha sido registrada en diversos cultivos de importancia económica en México (Arredondo y Rodríguez, 2008; 2015) y es considerado como uno de los depredadores de áfidos más importantes en la citricultura mexicana, al ejercer un control natural sobre las poblaciones de Aphis spiraecola Patch, Aphis gossypii Glover, Toxoptera citricida Kirkaldy y Toxoptera aurantii Boyer de Fonscolombe, vectores del Virus de la Tristeza de los Cítricos (VTC) (SENASICA, 2013).

A pesar de ser una especie común asociada a los cultivos agrícolas, el estudio sobre su biología y comportamiento como agente de control biológico, ha sido poco explorado en el estado de Nayarit. Rodríguez et al. (2015a) mencionaron que $C$. sanguinea es uno de los insectos depredadores de mayor abundancia en el cultivo de yaca (Artocarpus heterophyllus Lam.), y su incremento coincidió con los puntos máximos de infestación del pulgón $A$. aurantii sobre hojas, brotes tiernos e inflorescencias. La importancia de A. aurantii, además de su destacado potencial vectorial que ejerce en cítricos, es la capacidad de transmisión del hongo Rhizopus stolonifer (Ehrenb.: Fr.) Vuill, agente causal de la pudrición blanda en inflorescencias masculinas y femeninas de yaca, lo cual, en altas densidades, afecta la producción y comercialización de frutos en fresco en el mercado nacional e internacional (Rodríguez et al., 2017a; Medina et al., 2018).

Dada la importancia que podría desempeñar C. sanguinea como enemigo natural de $A$. aurantii en el cultivo de yaca, y en la búsqueda de establecer e implementar estrategias de control más sostenibles sobre esta plaga, el objetivo del presente trabajo fue determinar los aspectos biológicos y poblacionales de $C$. sanguinea sobre el pulgón $A$. aurantii en condiciones de laboratorio en el estado de Nayarit, México.

\section{MATERIALES Y MÉTODOS}

La investigación se realizó durante el periodo de junio a diciembre de 2017 en el Laboratorio de Parasitología Agrícola del Centro Multidisciplinario de Investigación Científica No. 3 (CEMIC 3) de la Universidad Autónoma de Nayarit (UAN), ubicado en el municipio de Tepic, Nayarit, México. En la recolección del material biológico se consideraron las zonas de mayor producción del cultivo de yaca en el Estado. La primera de ellas ubicada en El Llano, municipio de San Blas, Nayarit, México, localizada a $21^{\circ} 25^{\prime} 02,59^{\prime \prime}$ N y $105^{\circ} 11^{\prime} 19,89^{\prime \prime}$ O, y la segunda en Las Varas, municipio de Compostela, Nayarit, México, ubicada a $21^{\circ} 10^{\prime} 34,94^{\prime \prime}$ N y $105^{\circ} 10^{\prime} 12,44^{\prime \prime}$ O con una altitud de 30 y 20 msnm, respectivamente.

\section{Obtención y mantenimiento de Aphis aurantii}

Los áfidos se recolectaron de manera directa en los árboles de yaca en las zonas de producción anteriormente descritas. Para el establecimiento de la cría, bajo condiciones de invernadero de la UAN, la presa se mantuvo en plantas jóvenes de yaca (seis meses de edad) para asegurar su disponibilidad permanente. Para esto, con ayuda de un pincel se seleccionaron ninfas del $4^{\text {to }}$ ínstar y adultos de $A$. aurantii para infestar las hojas de las plantas, previamente colocadas en macetas de $20 \mathrm{~cm}$ de diámetro por 30 de altura, disponiendo del mismo sustrato de la huerta considerada para este estudio, y proporcionando riego diario con 
un atomizador (1 L). Las macetas se colocaron en jaulas entomológicas de $400 \times 120 \times 80 \mathrm{~cm}$, con marco de madera cubierta por tela de organza para permitir el manejo y ventilación de las mismas.

\section{Cría de Cycloneda sanguinea}

Los adultos de $C$. sanguinea se recolectaron del mismo sitio de muestreo que el pulgón. Los coccinélidos se depositaron en cajas de plástico transparente $(14 \times 14 \times 4,5 \mathrm{~cm})$ marca Inix ${ }^{\circledR}$ perforadas con un alfiler para permitir el intercambio gaseoso. En el interior de las cajas se aplicó miel en forma de líneas para proveer de alimento a los adultos del depredador. Una vez finalizada la recolecta, las muestras se trasladaron al CEMIC. En laboratorio se conformaron diez parejas, cada una de las cuales se colocaron en envases de plástico transparentes de un litro de capacidad, reemplazando su tapa por una cubierta de tela de organza sostenida con una banda elástica.

La alimentación del depredador se realizó diariamente utilizando hojas y brotes tiernos de yaca infestados con $A$. aurantii, las cuales sirvieron como sitio de oviposición. Se proporcionó agua a los depredadores mediante un trozo de algodón colocado en un frasco de vidrio de $25 \mathrm{~mL}$. Como alimento adicional para los adultos se colocó una toalla de algodón $\left(10 \mathrm{~cm}^{2}\right)$ humedecida con la mezcla de agua + miel de abeja + levadura de cerveza en una proporción 1:2:2. Los coccinélidos se colocaron en una cámara bioclimática (Thermo Scientific, Modelo No. 844, Dubuque, Iowa, E.U.A.) a una temperatura de $25 \pm 2^{\circ} \mathrm{C}, 70 \pm 10 \%$ de humedad relativa y 12 horas de fotofase, hasta obtener suficientes masas de huevecillos para iniciar los estudios biológicos.

\section{Ciclo de vida de $C$. sanguinea}

El ciclo biológico se determinó a partir de los individuos de la $\mathrm{F}_{1}$ obtenida en laboratorio. Las hojas o tejido vegetal ovipositadas por las hembras se aislaron en cajas de Petri de $90 \mathrm{~mm}$ de diámetro hasta su eclosión. Previamente las tapas de las cajas se sustituyeron por tela de organza para permitir la circulación de aire a los huevecillos. Cada caja se rotuló con una etiqueta indicando la especie del depredador, la presa y la fecha de oviposición. Se realizaron dos observaciones diarias (10:00 y 18:00 horas) para registrar el tiempo de incubación.

Una vez emergidas las larvas, se procedió a seleccionar 100 especímenes de manera aleatoria, los cuales se colocaron de manera individual en cajas de Petri de $60 \mathrm{~mm}$ de diámetro. Se realizaron observaciones diarias para registrar los eventos de ecdisis y muda para cada organismo. En cada ocasión se retiró la exuvia para facilitar el re- conocimiento de cada estado larval hasta emerger el adulto. Las larvas se alimentaron diariamente utilizando hojas de yaca infestadas de A. aurantii.

Para determinar la longevidad se seleccionaron al azar 30 adultos recién emergidos durante el estudio de ciclo de vida. Previamente, cada individuo fue sexado de acuerdo a las características mencionadas por Gordon (1985). De manera individual cada ejemplar se colocó en recipientes de plástico de $500 \mathrm{~cm}^{3}$ de capacidad, reemplazando su tapa por tela de organza para permitir el intercambio gaseoso. Los depredadores diariamente se alimentaron ad libitum con ninfas del pulgón $A$. aurantii hasta su muerte natural. Los recipientes se rotularon con una etiqueta indicando la especie del depredador, la fecha de emergencia, la especie de la presa, el sexo y número de réplica. El número de días que cada individuo permaneció vivo se registró para determinar la longevidad de los machos y hembras de C. sanguinea.

\section{Fecundidad y tasa de oviposición}

Se consideraron a los adultos de C. sanguinea con un tiempo de $8 \mathrm{~h}$ de emergencia y se formaron 10 parejas, cada pareja se colocó en cajas de Petri (90 mm de diámetro) por un periodo de 48 $\mathrm{h}$ para propiciar múltiples cópulas y favorecer la fecundidad de las hembras. Durante ese tiempo, los coccinélidos se alimentaron ad libitum con ninfas de $A$. aurantii. Se hizo seguimiento durante 14 días a partir de que la hembra ovipositó por primera vez, lo cual permitió comparar la capacidad reproductiva. El día en que las hembras empezaron a ovipositar luego de haber eclosionado como adultos, se consideró como el periodo de preoviposición. Se registró el total de huevos/hembra y huevos/hembra/día para estimar la fecundidad y tasa de oviposición. Los huevos recién ovipositados se depositaron cuidadosamente en cajas Petri de $90 \mathrm{~mm}$ de diámetro para ser incubados en la misma cámara de crianza. Esto permitió estimar el número de huevos que eclosionaron del total de huevos ovipositados por cada hembra (fertilidad).

\section{Tabla de vida y parámetros poblacionales de $C$. sanguinea}

La tabla de vida se determinó siguiendo la metodología de Rabinovich (1980), a partir de la edad específica de supervivencia $\left(l_{x}\right)$ y la edad específica de fecundidad $\left(m_{x}\right)$. Estos valores se obtuvieron de la información registrada en los experimentos de ciclo de vida y fecundidad anteriormente descritos. Además, se especificó para cada intervalo de edad los siguientes parámetros biológicos: Tasa neta de reproducción $\left(R_{0}\right)$, tasa intrínseca de crecimiento $\left(r_{m}\right)$, tasa finita de crecimiento $(\lambda)$ y tiempo generacional $(T)$. 


\section{Análisis estadístico}

Los datos obtenidos fueron analizados con el paquete estadístico SAS (Statistical Analysis System) para Windows ${ }^{\circledR}$ Versión 9.0. Para determinar la diferencia en la longevidad entre sexos, se aplicó una prueba t-Student para varianzas homogéneas. La capacidad reproductiva se estimó mediante análisis de varianza (ANOVA) y prueba de comparación de medias LSD $(a=0,05)$. Los valores de la tabla de vida se analizaron a través del método Life-Table del programa SAS.

\section{RESULTADOS Y DISCUSIÓN}

\section{Ciclo de vida de $C$. sanguinea}

La duración promedio de la fase inmadura de $C$. sanguinea alimentado con $A$. aurantii fue de 19,32 $\pm 4,97$ días (Tabla 1). Estos resultados son similares a los registrados por Cardoso y Lázzari (2003) y Rodríguez et al. (2015b), quienes determinaron que el desarrollo de huevo hasta la emergencia de los adultos de $C$. sanguinea sobre el pulgón Cinara spp. y Toxoptera citricida Kirkaldy, fue de 17,5 y 19,2 días, respectivamente. Sin embargo, difieren con lo reportado por Solano et al. (2016) al obtener una duración media de 11,8 días alimentado con el pulgón Aphis craccivora Koch en las mismas condiciones de temperatura $\left(25^{\circ} \mathrm{C}\right)$. Por otra parte, Rodríguez et al. (2016) registraron una duración de 16,6 días sobre $A$. aurantii a una temperatura de $26 \pm 3^{\circ} \mathrm{C}$.

El desarrollo embrionario de $C$. sanguinea fue de 3,78 $\pm 0,46$ días. Estos resultados superan a lo registrado por Rodríguez et al. (2016) quienes reportaron una duración de 2,42 días para la fase de huevo, mientras que Rodríguez et al. (2015b) registraron una duración de 3,76 días. De acuerdo a Omkar y Bind (2004), Omkar y James (2004) y a Omkar y Pervez (2004), la diferencia en el periodo de incubación de huevo para coccinélidos alimentados con diferentes especies de áfidos se debe al valor nutritivo de los pulgones y a la temperatura de la cría.

El periodo de la fase larval fue de 10,34 $\pm 2,49$ días, siendo el cuarto instar el de mayor duración (3,25 $\pm 0,66$ días). Estos resultados coinciden con lo registrado por Santa et al. (2001), Cardoso y Lázzari (2003), Oliveira et al. (2004), Rodríguez et al. (2015b) y Rodríguez et al. (2016) para el mismo coccinélido. Machado (1982) señaló que el cuarto instar es el de mayor duración debido a que el coccinélido debe suplir las necesidades nutritivas exigidas para la transformación en pupa y posterior emergencia de los adultos.

En prepupa, C. sanguinea tuvo una duración de $1,74 \pm 0,67$ días, lo cual supera a lo reportado por Veloso et al. (1995) y Santa et al. (2001) quienes indicaron una duración de 0,86 y un día, respectivamente. El estado de pupa presentó una duración de 3,46 $\pm 1,35$ días, lo cual coincide con lo reportado por Rodríguez et al. (2016) quienes registraron una duración promedio de 3,45 días.

González (2006) mencionó que el ciclo de vida de los coccinélidos varía de acuerdo al tipo de presa consumida y a las condiciones de temperatura en las que se encuentre, por lo que ambas

Tabla 1. Duración media en días del ciclo de vida de Cycloneda sanguinea L. sobre Aphis aurantii Boyer de Fonscolombe en Nayarit, México.

Table 1. Mean duration (days) of the life cycle of Cycloneda sanguinea L. on the aphid Aphis aurantii Boyer de Fonscolombe in Nayarit, Mexico.

\begin{tabular}{lcc}
\hline $\begin{array}{l}\text { Estado de } \\
\text { desarrollo }\end{array}$ & $\begin{array}{c}\text { Número de individuos } \\
\text { evaluados }\end{array}$ & Duración en días \\
\cline { 3 - 3 } Huevo & 100 & Media \pm DE \\
L1 & 100 & $3,78 \pm 0,46$ \\
L2 & 100 & $2,52 \pm 0,55$ \\
L3 & 100 & $2,38 \pm 0,64$ \\
L4 & 99 & $2,19 \pm 0,62$ \\
Prepupa & 87 & $3,25 \pm 0,66$ \\
Pupa & 87 & $1,74 \pm 0,67$ \\
Huevo-Adulto & - & $3,46 \pm 1,35$ \\
Machos & 16 & $19,32 \pm 4,97$ \\
Hembras & 14 & $58,37 \pm 3,44$ a \\
\hline
\end{tabular}

Los valores promedio con diferentes letras difieren estadísticamente (t de Student, para varianzas homogéneas $\mathrm{P}<0,05$ ).

Mean values with different letters differ statistically (Student's t-test for homogeneous variances test $\mathrm{P}<0.05)$. 
variables tuvieron un efecto significativo en el tiempo de desarrollo de $C$. sanguinea alimentado con el pulgón $A$. aurantii.

\section{Longevidad y proporción sexual de $C$. sanguinea}

La longevidad promedio de los adultos tuvo una duración de 59,93 $\pm 8,41$ días a una temperatura de $25^{\circ} \mathrm{C}$. La longevidad de las hembras $(61,71$ $\pm 4,96$ días) fue superior a la de los machos (58,37 $\pm 3,44)$, y de acuerdo a los datos obtenidos mediante la prueba de $t(t=2,16, \mathrm{gl}=28, P=0,0393)$, existen diferencias estadísticamente significativas entre los sexos (Tabla 1). Estos resultados son similares a los reportados por Santos y Queiroz (1981), quienes registraron una longevidad promedio de 63 días para adultos de C. sanguinea sobre $T$. aurantii, a una temperatura de $25^{\circ} \mathrm{C}$. Solano et al. (2016) utilizaron como presa al pulgón $A$. craccivora y registraron una longevidad de 83,10 $\pm 9,8$ y $83,8 \pm 11,4$ días para machos y hembras, respectivamente, bajo las mismas condiciones de temperatura. Por otra parte, Cardoso y Lázzari (2003) reportaron una longevidad de 167,1 días sobre Cinara spp., mientras que Oliveira et al. (2004) obtuvieron una duración promedio de 125,7 días para adultos de C. sanguinea alimentados con Cinara atlantica Wilson a una temperatura de 20 y $24^{\circ} \mathrm{C}$, respectivamente.

En lo que respecta a la proporción de sexos, se encontró una relación macho:hembra de 1:0,87 sobre la presa evaluada. Datos similares fueron reportados por Solano et al. (2016) con una proporción de 1:0,76 para machos y hembras de $C$. sanguinea alimentados con el pulgón $A$. craccivora. Oliveira et al. (2004) reportaron una relación de 1:1, 1:1,17 y 1:1,17 para los coccinélidos Hippodamia convergens Guérin-Meneville, $C$. sanguinea y Eriopis connexa (Germar), respectivamente.

\section{Fecundidad y tasa de oviposición de $C$. sangui- nea}

El periodo de pre-oviposición de las hembras de C. sanguinea a una temperatura de $25^{\circ} \mathrm{C}$ fue de $3,9 \pm 0,31$ días. Dos Santos et al. (2013) registraron valores similares de pre-oviposición $(3,8 \pm 0,37)$ para C. sanguinea alimentado con Schizaphis graminum (Rondani). Sin embargo, estos resultados difieren con lo reportado por Solano et al. (2016), quienes obtuvieron una pre-oviposición promedio de 6,0 $\pm 1,1$ días para $C$. sanguinea sobre $A$. craccivora en las mismas condiciones de temperatura. Por otra parte, Milléo et al. (2014) indicaron una pre-oviposición de 24,30 $\pm 1,36,9,50 \pm 0,42$ y $12,20 \pm 8,89$ días para las especies $C$. sanguinea, Harmonia axyridis Pallas y $H$. convergens, respectivamente, alimentadas con Anagasta kuehniella Zeller. Zhao et al. (2015) registraron un periodo de pre-oviposición de 8,8 \pm 0,3 días para el coc- cinélido Cheilomenes sexmaculata Fabricius sobre M. persicae; mientras que Ghanim et al. (2014) reportaron un promedio de 4,0 a 5,0 y 5,0 a 5,5 días de pre-oviposición en hembras de Coccinella undecimpunctata Linnaeus alimentadas con Sitobion avenae Fabricius y S. graminum, respectivamente.

El promedio de huevos que colocó cada hembra durante toda su vida fue de $882,2 \pm 52,28$, mientras que la tasa de oviposición registrada fue de 34,6 6 0,51 huevos/hembra/día; estos resultados son similares a lo reportado por Omkar y James (2004) al registrar una fecundidad de 874 \pm 45 ,16 huevos/hembra, y una tasa media reproductiva de 19 huevos/día/hembra para Coccinella transversalis Fabricius sobre Lipaphis erysimi Kaltenbach a una temperatura de $27^{\circ} \mathrm{C}$, pero difiere al ser alimentado con otras especies de áfidos como A. gossypii, A. craccivora, Aphis nerii Boyer, M. persicae y Uroleucon compositae Theobald. Dos Santos et al. (2013) determinaron valores de $1.021 \pm 76,69,1.029,2 \pm 228,6$ y $484,5 \pm 35,82$ huevos/hembra para los coccinélidos $C$. sanguinea, $H$. axyridis e $H$. convergens, respectivamente, alimentados con $S$. graminum a una temperatura de $25^{\circ} \mathrm{C}$; mientras que Castro et al. (2011) registraron una variación significativa en la fecundidad de las hembras de $H$. axyridis sobre $C$. atlantica bajo diferentes condiciones de temperatura.

De acuerdo a Evans (2003) la calidad nutricional y disponibilidad de la presa, así como la temperatura de la cría, determinan la fecundidad de los coccinélidos, de manera que las hembras de C. sanguinea evaluadas en este estudio, tendieron a ovipositar las suficientes masas de huevecillos (bajo las condiciones de temperatura establecidas y la cantidad de presas disponibles) para asegurar su descendencia y posterior supervivencia.

\section{Tabla de vida de $C$. sanguinea}

La curva de sobrevivencia de $C$. sanguinea registró un descenso entre los días 15 y 20 durante el estado de prepupa y pupa, periodo en el que comenzó a declinar progresivamente el número de individuos vivos (Fig. 1). Este comportamiento refleja una curva de tipo I, en donde la mortalidad se concentra principalmente en los individuos de edades avanzadas (Rabinovich, 1980).

Estos resultados coinciden con los registrados por Milléo et al. (2014) y Solano et al. (2016) quienes encontraron una curva de tipo I para $C$. sanguinea alimentado con huevos de A. kuehniella y ninfas de $A$. craccivora, respectivamente, en las mismas condiciones de temperatura evaluadas en este estudio. Por otra parte, diversos autores registraron un comportamiento similar en otras especies de coccinélidos como Cryptolaemus montrouzieri Mulsant, Coccinella septempunctata L., Ceratomegilla undecimnotata Schneider, Propylea 


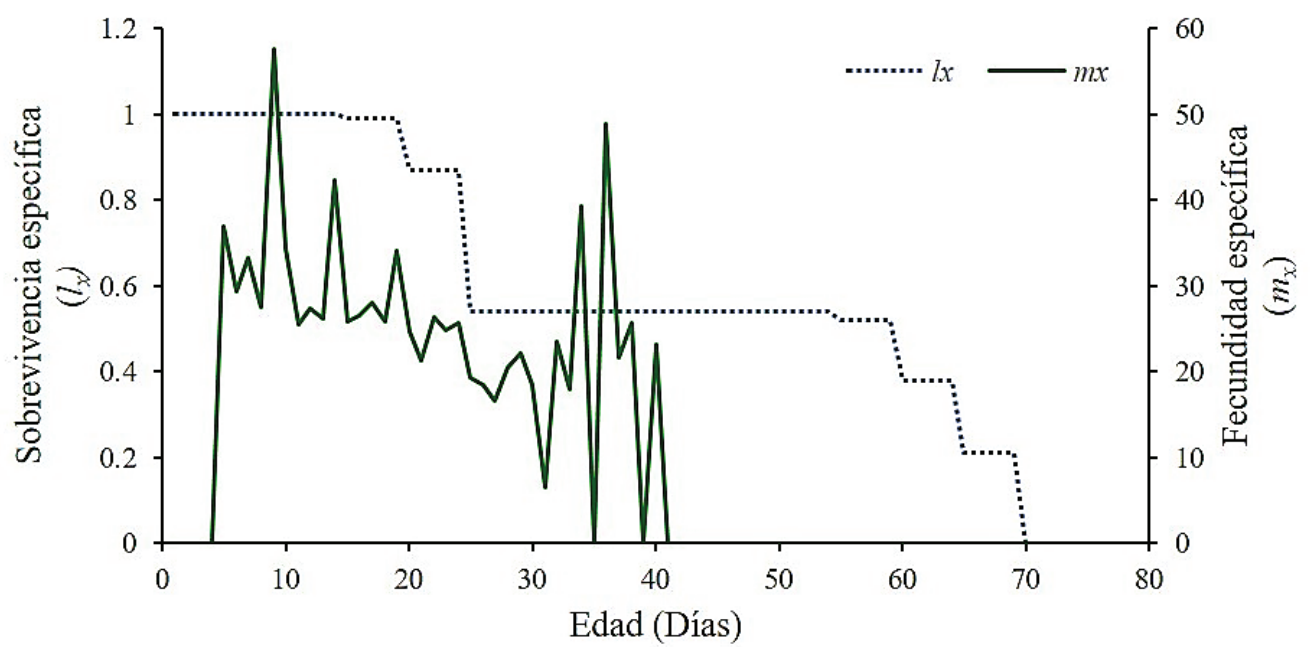

Fig. 1. Curva de sobrevivencia específica por edades $\left(l_{x}\right)$ y curva de fecundidad específica $\left(m_{x}\right)$ de Cycloneda sanguinea L. sobre Aphis aurantii Boyer de Fonscolombe en condiciones de laboratorio.

Fig. 1. Age-specific survival curve $\left(l_{x}\right)$ and specific fertility curve $\left(m_{x}\right)$ of Cycloneda sanguinea L. on Aphis aurantii Boyer de Fonscolombe under laboratory conditions.

quatuordecimpunctata L., E. connexa, H. axyridis y Exochomus marginipennis LeConte (Torres y Marcano, 2007; Kontodimas et al., 2008; Duarte y Zenner, 2009; Ali y Rizvi, 2010; Castro et al., 2011; Palomares et al., 2016).

Rabinovich (1980) mencionó que las curvas de sobrevivencia son una forma de expresar la mortalidad a la que está sujeta una población, y varía en función de las condiciones ambientales, al sexo, al genotipo de los individuos y al papel ecológico que ejercen dentro de la comunidad en que viven; por lo que la mortalidad de $C$. sanguinea en este trabajo se vio afectada por las condiciones de temperatura descritas anteriormente, y por agentes bióticos como el parasitoide Dinocampus coccinellae Schrank (Hymenoptera: Braconidae), el cual se desarrolla como endoparasitoide de coccinélidos adultos (Rodríguez et al., 2017b), y por patógenos no identificados que redujeron la población de los individuos en estado de pupa.

La curva de fecundidad $\left(m_{x}\right)$ de $C$. sanguinea mostró una variación constante en la oviposición de las hembras durante el periodo de estudio (Fig. 1), se logró observar dos punto máximos en los días 9 y 36 después de la emergencia de los adultos. Castro et al. (2011) registraron un comportamiento similar en las hembras de $H$. axyridis sometidas a diferentes temperaturas $(15,20 \mathrm{y}$ $25^{\circ} \mathrm{C}$ ) y alimentadas con ninfas de C. atlantica en condiciones de laboratorio; mientras que Solano et al. (2016) reportaron dos puntos máximos de oviposición en los días 30 y 35 para C. sanguinea.

Respecto a los parámetros reproductivos, el valor estimado de la tasa neta de reproducción $\left(R_{0}\right)$ de $C$. sanguinea fue de 705,76 huevos hembra/ hembra, en un tiempo generacional $(T)$ de 23,39 días y una tasa finita de crecimiento $(\lambda)$ de 1,41 individuos por día (Tabla 2). Es decir, en las condiciones de temperatura consideradas en este estudio, cada hembra de $C$. sanguinea es reemplazada por 705 hembras hijas al cabo de una generación; mientras que el tiempo generacional registrado, determina que a partir de que un huevo es puesto, se requieren 23 días para que una hembra, proveniente del mismo, inicie su reproducción. Los resultados son similares a los reportados por Solano et al. (2016), quienes indicaron una tasa de reemplazo de 718,79 hembras/hembra en un tiempo generacional de 35 días; pero difieren con Zazycki et al. (2015) al registrar tasas de reproducción bajas para los coccinélidos E. connexa, $H$. axyridis y Olla v-nigrum Mulsant alimentados con huevos de A. kuehniella. Por otra parte, Kontodimas et al. (2008) obtuvieron valores altos en la tasa neta de reproducción (1.004,1 hembras/ hembra) y un tiempo generacional de 58,6 días para C. septempunctata sobre Aphis fabae Scopoli; mientras que Milléo et al. (2014) registraron una tasa de 2.226,89 hembras/hembra en un tiempo de 55,49 días para los individuos de $H$. axyridis alimentados con A. kuehniella.

La estimación de la tasa intrínseca de crecimiento $\left(r_{m}\right)$ proporcionó un valor de 0,17 , es decir, que la población de $C$. sanguinea tiene un crecimiento aproximado al $20 \%$ del total de los individuos por unidad de tiempo. Estos valores 
Tabla 2. Parámetros poblacionales de Cycloneda sanguinea L. sobre Aphis aurantii Boyer de Fonscolombe en condiciones de laboratorio en Nayarit, México.

Table 2. Population parameters of Cycloneda sanguinea L. on Aphis aurantii Boyer de Fonscolombe under laboratory conditions in Nayarit, Mexico.

\begin{tabular}{lc}
\hline Parámetros & Valores \\
\hline Tasa neta de reproducción $\left(R_{0}\right)$ & 705,76 \\
Tasa intrínseca de crecimiento $\left(r_{m}\right)$ & 0,17 \\
Tasa finita de crecimiento $(\lambda)$ & 1,41 \\
Tiempo generacional $(T)$ & 23,39 \\
\hline
\end{tabular}

se asemejan a los obtenidos por Zhao et al. (2015), quienes registraron una tasa de crecimiento de $0,16 \pm 0,0062$ para C. sexmaculata alimentada con M. persicae.

De acuerdo con García et al. (2005), los factores que afectan el equilibrio de una población en condiciones de laboratorio, están sujetos principalmente por la tasa de mortalidad, la curva de sobrevivencia y los parámetros de reproducción de los individuos, por lo que éstas variables determinan que la dinámica poblacional observada sirve de partida para predecir las posibles interacciones que existen entre $C$. sanguinea y el pulgón A. aurantii bajo condiciones de campo.

\section{CONCLUSIONES}

Los datos biológicos (ciclo de vida) y los parámetros poblacionales (curva de sobrevivencia y fecundidad) obtenidos en este estudio, son atributos que determinan la efectividad de los enemigos naturales y la integración de éstos dentro de los programas de control biológico. Por su rápido desarrollo y alta capacidad reproductiva, Cycloneda sanguinea podría ser considerado como un depredador potencial para el control de Aphis aurantii en el cultivo de yaca.

\section{AGRADECIMIENTOS}

A la Universidad Autónoma de Nayarit y al Consejo Nacional de Ciencia y Tecnología (CONACyT). Al Posgrado en Ciencias Biológico, Agropecuarias de la UAN.

\section{LITERATURA CITADA}

Ali, A., and P.Q. Rizvi. 2010. Age and stage specific life table of Coccinella septemounctata (Coleoptera: Coccinellidae) at varying temperature. World Journal of Agricultural Sciences 6(3):268-273.

Arredondo, B.H.C., y L.A. Rodríguez. 2008. Casos de Control Biológico en México. $1^{\text {a }}$ ed. Mundi Prensa México, S.A. de C.V., México, D.F.
Arredondo, B.H.C., y L.A. Rodríguez. 2015. Casos de control biológico en México. Vol. 2. Printing Arts México, S. de R.L. de C.V. Guadalajara, Jalisco, México.

Cardoso, J.T., and S.M. Lázzari. 2003. Comparative biology of Cycloneda sanguinea (Linnaeus, 1763) and Hippodamia convergens Guerin-Meneville, 1842 (Coleoptera: Coccinellidae) focusing on the control of Cinara spp. (Hemiptera: Aphididae). Revista Brasileira de Entomología 47(3):443-446.

Castro, C.F., L.M. Almeida, and S.R.C. Penteado. 2011. The impact of temperature on biological aspects and life table of Harmonia axyridis (Pallas) (Coleoptera: Coccinellidae). Florida Entomologist 94(4):923-932.

Castro, T.R.E. 2017. Familia Coccinellidae. p. 260262. En T.D. Cibrián (ed.). Fundamentos de Entomología Forestal. Universidad Autónoma Chapingo, Texcoco, Estado de México, México.

Dos Santos, L.C., T.M. Dos Santos, F.J. Cividanes, and S.T. Soares. 2013. Biological aspects of Harmonia axyridis in comparison with $\mathrm{Cy}$ cloneda sanguinea and Hippodamia convergens. Revista Pesquisa Agropecuária Brasileira 48(11):1419-1425.

Duarte, G.W., e I. Zenner. 2009. Tabla de vida del cucarrón depredador Eriopis connexa connexa (Germar) (Coleoptera: Coccinellidae). Revista U.D.C.A Actualidad y Divulgación Científica 12(2):147-155.

Evans, E.W. 2003. Searching and reproductive behaviour of female aphidophagous ladybirds (Coleoptera: Coccinellidae): a review. European Journal of Entomology 100:1-10.

Funichello, M., L.L. Costa, O.J. Aguirre, y A.C. Busoli. 2012. Aspectos biológicos de Cycloneda sanguinea (Coleoptera: Coccinellidae) alimentadas con pulgones criados en algodón transgénico Bollgard I. Revista Colombiana de Entomología 38(1):156-161. 
García, G. J., E.R. Benítez, y A. López. 2005. Tabla de vida de Delphastus pusillus (Coleoptera: Coccinellidae) en la mosca blanca Trialeurodes vaporariorum (Hemiptera: Aleyrodidae). Revista Colombiana de Entomología 31(2):155160.

Ghanim, A.A., F.E. Abd, and A.A. Abd. 2014. Influence of nutrition's on longevity and fecundity the coccinellid predators, Coccinella undecimpunctata L. (Coleoptera: Coccinellidae). Journal of Plant Protection and Pathology 5(7):827-835.

González, G. 2006. Los Coccinellidae de Chile. Disponible en: https://www.coccinellidae.cl/ index.php. (Consulta 12 junio 2018).

Gordon, R.D. 1985. The Coccinellidae (Coleoptera) of America North of Mexico. Journal of the New York Entomological Society 93(1):1912.

Kontodimas, D.C., P.G. Milonas, G.J. Stathas, N.E. Papanikolou, A. Skourti, and Y.G. Matsinos. 2008. Life table parameters of the aphid predators Coccinella septempunctata, Ceratomegilla undecimnotata and Propylea quatuordecimpunctata (Coleoptera: Coccinellidae). European Journal of Entomology 105:427-430.

López, A.J.I., E. Cortez, H.C. Arredondo, M. Ramírez, J. Loera, y M.A. Mellín. 2007. Uso de artrópodos depredadores para el control biológico de plagas en México. p. 90-105. En L.A. Rodríguez, y H.C. Arredondo (eds.). Teoría y Aplicación del Control Biológico. Sociedad Mexicana de Control Biológico, México, D.F.

Machado, V.L.R. 1982. Morfologia e aspectos biologicos de Cycloneda conjugata Mulsant, 1866 e Olla v-nigrum (Mulsant, 1866) (Coleoptera, Coccinellidae) predadores de Psylla sp. (Homoptera, Psyllidae) em sibipiruna (Caesalpinia pelthophoroides Benth.). Dissertação de Mestrado. Escola Superior de Agricultura Luiz de Queiroz, Universidade de São Paulo, Brasil.

Medina, T.M.A., G. Luna, J. Cambero, L.G. Ramírez, y C. Ríos. 2018. Agentes causales de la pudrición de inflorescencias de Artocarpus heterophyllus y asociación con Toxoptera aurantii en Nayarit, México. Revista Bio Ciencias 5(2):e438. doi: 10.15741/revbio.05.02.07.

Milléo, J., F. Sales, and W.A. Conde. 2014. Comparative demography of the exotic Harmonia axyridis with other aphidophagous coccinellids reared on artificial diet. Revista Pesquisa Agropecuária Brasileira 49(1):1-10.
Oliveira, N.C., C.F. Wilcken, y C.A. O. de Matos. 2004. Ciclo biológico y depredación de tres especies de coccinélidos (Col.: Coccinellidae) sobre el pulgón gigante del pino Cinara atlantica (Wilson) (Hemiptera: Aphididae). Revista Brasileira de Entomología 48(4):529-533.

Omkar, O., and R.B. Bind. 2004. Prey quality dependent growth, development and reproduction of a biocontrol agent, Cheilomenes sexmaculata (Fabricius) (Coleoptera: Coccinellidae). BioControl Science and Technology 14(7):665-673.

Omkar, O., and B.E. James. 2004. Influence of prey species on immature survival, development, predation and reproduction of Coccinella transversalis Fabricius (Coleoptera: Coccinellidae). Journal of Applied Entomology 128:150-157.

Omkar, O., and A. Pervez. 2004. Functional and numerical responses of Propylea dissecta (Coleoptera: Coccinellidae). Journal of Applied Entomology 128:140-146.

Palomares, P.M., B. Rodríguez, M.A. Ayala, J.J. De la Cruz, A.M. Mendoza, J.A. Sánchez, et al. 2016. Aspectos biológicos y capacidad de depredación de Exochomus marginipennis (Leconte) (Coleoptera: Coccinellidae) sobre Diaphorina citri Kuwayama (Hemiptera: Liviidae). Chilean Journal of Agricultural \& Animal Science 32(2):102-109.

Rabinovich, J.E. 1980. Introducción a la Ecología de Poblaciones Animales. Compañía Editorial Continental, México, D.F.

Rodríguez, P.M., J. Cambero, G. Luna, A. Robles, C. Carvajal, y C. Santillán. 2015a. Fluctuación poblacional y especies de coccinélidos (Coleoptera: Coccinellidae) asociadas al cultivo de yaca (Artocarpus heterophyllus Lam.) en Nayarit, México. Entomología Mexicana 2(2):306-311.

Rodríguez, P.M., J. Cambero, C. Carvajal, O. Estrada, y N. De Dios. 2015b. Ciclo de vida de Cycloneda sanguinea Linnaeus (Coleoptera: Coccinellidae) sobre el pulgón café de los cítricos Toxoptera citricida Kirkaldy (Hemiptera: Aphididae) en Nayarit, México. p. 191-196. En XXXVIII Congreso Nacional de Control Biológico. 5-6 de Noviembre. León, Guanajuato, México. Sociedad Mexicana de Control Biológico, Texcoco, Estado de México, México. 
Rodríguez, P.M., J. Cambero, G. Luna, A. Robles, C.L. Yáñez, y K.G. Cambero. 2016. Ciclo de vida de Cycloneda sanguinea Linnaeus (Coleoptera: Coccinellidae) sobre el pulgón Aphis aurantii Boyer de Fonscolombe (Hemiptera: Aphididae) en Nayarit, México. En XXXIX Congreso Nacional de Control Biológico. 7-9 de Noviembre. Guadalajara, Jalisco, México. Sociedad Mexicana de Control Biológico, Texcoco, Estado de México, México.

Rodríguez, P.M., J. Cambero, G. Luna, A. Robles, R. Peña, y A.L. Muñoz. 2017a. Primer registro de Aphis (Toxoptera) aurantii en Artocarpus heterophyllus Lam. (Moraceae) en México. Southwestern Entomologist 42(4):1111-1114.

Rodríguez, P.M., J. Cambero, N. De Dios, y K.G. Cambero. 2017b. Primer registro de Dinocampus coccinellae (Schrank) (Hymenoptera: Braconidae) como parasitoide de Cycloneda sanguinea Linnaeus (Coleoptera: Coccinellidae) en México. Folia Entomológica Mexicana (n.s.) 3(1):12-14.

Santa, C.L.V.C., R.C.R. Goncalves, R.M. Sales, y F.R. Do Nascimento. 2001. Aspectos biológicos e consumo de larvas de Cycloneda sanguinea (Linnaeus, 1763) (Coleoptera: Coccinellidae) alimentadas con Schizaphis graminum (Rondani, 1852) (Hemiptera: Aphididae). Ciência e Agrotecnología Lavras 25(6):12731278.

Santos, G.P., y A.C. Queiroz. 1981. Biologia de Cycloneda sanguinea e sua associação com pulgão em mudas de mangueira. Pesquisa Agropecuária Brasileira 16(4):473-76.

SENASICA. 2013.Pulgón café de los cítricos (Toxoptera citricida Kirkaldy). Dirección General de Sanidad Vegetal-Sistema Nacional de Vigilancia Epidemiológica Fitosanitaria. Ficha Técnica No. 37. Servicio Nacional de Sanidad, Inocuidad y Calidad Agroalimentaria (SENASICA), México, D.F.
Solano, Y., N. Delgado, J. Morales, and C. Vázquez. 2016. Biological studies and life table of Cycloneda sanguinea (L.) (Coleoptera: Coccinellidae) on Aphis craccivora Koch (Hemiptera: Aphididae). Entomotropica 31(34):267275.

Torres, F., y R. Marcano. 2007. Efecto de la temperatura en el desarrollo de Cryptolaemus montrouzieri Mulsant (Col.: Coccinellidae) utilizando como presa Maconellicoccus hirsutus Green (Hemiptera: Pseudococcidae). Entomotropica 22(1):17-25.

Vandenberg, N. 2002. The new world genus Cycloneda Crotch (Coleoptera: Coccinellidae): Historical review, new diagnosis, new generic and specific synonyms, and an improved key to North American species. Proceedings of the Entomological Society of Washington 104(1):221-236.

Veloso, V.R.S., R. Veloso, J.L. Nascimento, P.M. Fernández, y A.H. García. 1995. Aspectos biológicos de Cycloneda sanguinea (L.) (Coleoptera: Coccinellidae). Anais das Escolas de Agronomía e de Veterinaria 25(2):123-127.

Zazycki, L.C.F., R.E.S. Semedo, A. Silva, A.Z. Bisognin, O. Bernardi, M.S. García, et al. 2015. Biology and fertility life table of Eriopis connexa, Harmonia axyridis and Olla v-nigrum (Coleoptera: Coccinellidae). Brazilian Journal of Biology 75(4):969-973.

Zhao, J., S. Li, X.W. Gao, F. Zhang, and S. Wan. 2015. Comparison of life tables of Cheilomenes sexmaculata (Coleoptera: Coccinellidae) under laboratory and greenhouse conditions. Journal of Economic Entomology 108(4):1700-1707. 